\title{
A discrete tracking-differentiator design for active vibration control in maglev systems
}

\author{
Zhi-Zhou Zhang ${ }^{1, \mathrm{a}, \dagger}$ and Yun-De Xie ${ }^{2, \mathrm{~b}}$ \\ ${ }^{1}$ College of Aerospace Science and Technology, National University of Defense \\ Technology, Changsha, Hunan, 410073, P. R. China \\ ${ }^{2}$ Changsha Branch, Beijing holdings Maglev Technology Development Co., Ltd., \\ Changsha 410073, P.R. China \\ ${ }^{a} z z z 336 @ 126 . c o m,{ }^{b} z z z 880 @$ hotmail.com \\ *Corresponding author
}

\begin{abstract}
A second-order linear differentiator with small phase-delay and amplitude attenuation based on a fast synthesis function is presented for active vibration control in maglev systems. The discrete linear structure of the differentiator is given and its tracking and filtering ability is analyzed in time domain. The restraining disturbance performance with optimal control theory for bounded disturbance is discussed.
\end{abstract}

Keywords: Second-order system; Linear differentiator; Signal filter; Bounded disturbance; Optimal control

\section{Introduction}

In engineering practice, continuous signal and its differential signal often needs to be drawn reasonably from the discontinuous signal with random noise. However, in the traditional control engineering, it is very difficult to acquire the differential signal because the pure differentiator is unable to realize in physics. Especially, if there are some random noises in the signal, the quality of the corresponding differential signal would have trouble, and even worse it may be submerged by magnified noise [1]. Therefore, designing a simple and practical differentiator has great significance to simplify the controller design and improve the controller performance.

Generally, we often use the approximation method to gain a similar differential signal. Some scholars [2-3] have proposed many ways to obtain differential signals and linear differentiator is the simplest one. Linear differentiator with high-gain can provide $\mathrm{n}-1$ order derivative, but this kind differentiator may contain disturbance directly in every level [4]. Linear derivative tracker has a faster convergence rate when system far from the equilibrium point, while close to equilibrium point the convergence rate will slow down [5]. The sliding mode differentiator with second order need to know in 
advance that the upper bound of Lipschitz constant for the signal derivative, thus limits the type of input signal, and the chattering is inevitable [6-7]. Paper [8] studies the optimal integrated functions for double integral series system, the introduction of the switching curve in the vicinity of the linear region, and with the precise control strategy a synthesis fast discrete function is acquired. Zhang D.[9], Li H.[10], Han J.Q. [11] design a band-stop filter, a skyhook damping filter and a gap differentiator respectively, to a certain extent, which can restrain the vehicle-guideway coupled vibration. Lingling Zhang [12] proposes a hybrid nonlinear gap differentiator with global fast convergence.

Using the fast synthesis function, this paper constructs a second order linear differentiator with small phase delay and amplitude attenuation, gives the discrete linear structure, analyzes its ability to track and filter in the time domain, and discusses the ability of boundary disturbance immunity of the system based on the optimal theory and singular perturbation technical.

\section{Second-Order Discrete Nonlinear Tracking Differentiator}

Consider a second-order double integral series of continuous system

$$
\left\{\begin{array}{l}
\dot{x}_{1}=x_{2} \\
\dot{x}_{2}=r u,|u| \leq 1
\end{array}\right.
$$

Suppose the initial state as $x_{1}(0)=x_{10}, x_{2}(0)=x_{20}$. According to the optimal control theory, continuous system (1) has an optimal control strategy that arbitrary point $M\left(x_{10}, x_{20}\right)$ in phase plane can achieve to the origin point at most one switch and the required time is the least.

Let $\Gamma\left(x_{1}(k), x_{2}(k), r\right)=x_{1}(k)+\frac{x_{2}(k) \cdot\left|x_{2}(k)\right|}{2 \cdot r}$, the set of switching points, that is the switch curve can be expressed as following:

$$
\Gamma\left(x_{1}, x_{2}, r\right)=x_{1}+\frac{1}{2 \cdot r} x_{2} \cdot\left|x_{2}\right|=0
$$

The optimal solution of the above control question is

$$
u(t)=\left\{\begin{array}{l}
-\operatorname{sign}\left(\Gamma\left(x_{1}, x_{2}, r\right)\right), \text { if } \Gamma\left(x_{1}, x_{2}, r\right) \neq 0 \\
\operatorname{sign}\left(x_{2}\right), \text { if } \Gamma\left(x_{1}, x_{2}, r\right)=0, x_{2} \neq 0
\end{array}\right.
$$

If $M\left(x_{10}, x_{20}\right)$ is not in the switch curve (2), by adopting the control law(3), the time $t_{A}$ that $M\left(x_{10}, x_{20}\right)$ reaches to the switch curve(2) is 


$$
t_{A}=s \cdot \frac{x_{2}}{r}+\sqrt{s \cdot \frac{x_{1}}{r}+\frac{1}{2} \frac{x_{2}^{2}}{r^{2}}}
$$

If $M\left(x_{10}, x_{20}\right)$ is in the switch curve (2), $\Gamma\left(x_{1}, x_{2}, r\right)=0$, by adopting the control law(3), the time $t_{B}$ that $M\left(x_{10}, x_{20}\right)$ reaches to the origin point $O$ is

$$
t_{B}=\frac{\left|x_{2}\right|}{r}
$$

In order to get the fast control function for second order discrete system which corresponds to system (1), paper [1] proposes an exact discrete control strategy that $M\left(x_{10}, x_{20}\right)$ in the phase plane reaches to the origin point after a series steps $(k=0,1,2 \cdots)$ with sample step $h$ by fixed step method.

$$
\left\{\begin{array}{l}
{[u(k), b]=f a s t\left(x_{1}(k), x_{2}(k), r, h\right)} \\
x_{1}(k+1)=x_{1}(k)+h \cdot x_{2}(k)+\frac{1}{2} r h^{2} \cdot u(k)-\frac{1}{3} b h^{3} \\
x_{2}(k+1)=x_{2}(k)+r h \cdot u(k)-\frac{1}{2} b h^{2}, k=0,1,2 \cdots
\end{array}\right.
$$

For system (1), the function $[u(k), b]=f a s t\left(x_{1}(k), x_{2}(k), r, h\right)$ can be sure by the following rules:

(1) When $\Gamma\left(x_{1}(k), x_{2}(k), r\right)>0$, let $s=\operatorname{sign}\left(\Gamma\left(x_{1}(k), x_{2}(k), r\right)\right)$, $t_{A}=\frac{x_{2}(k)}{r} \cdot s+\sqrt{\frac{x_{1}(k)}{r} \cdot s+\frac{x_{2}^{2}(k)}{2 \cdot r^{2}}}$, then

1)if $t_{A} \geq h$,then

$$
\left\{\begin{array}{l}
b=0 \\
u(k)=-s
\end{array}\right.
$$

2)if $t_{A}<h$, then

$$
\left\{\begin{array}{l}
b=0 \\
u(k)=-\left(-\frac{1}{2}+\frac{x_{2}(k) s}{r h}+\frac{1}{2} \sqrt{\left.1+\left(\frac{4 x_{2}(k)}{r h}+\frac{8 x_{1}(k)}{r h^{2}}\right) s\right) s}\right.
\end{array}\right.
$$

(2) When $\Gamma\left(x_{1}(k), x_{2}(k), r\right)=0$, let $t_{B}=\frac{\left|x_{2}(k)\right|}{r}$, then 1)if $t_{B} \geq h$, then 


$$
\left\{\begin{array}{l}
b=0 \\
u(k)=-\operatorname{sign}\left(x_{2}(k)\right)
\end{array}\right.
$$

2)if $t_{B}<h$, then

$$
\left\{\begin{array}{l}
b=\frac{6\left(x_{2}(k) h+2 x_{1}(k)\right)}{r h^{3}} \\
u(k)=-\frac{2\left(2 x_{2}(k) h+3 x_{1}(k)\right)}{r h^{2}}+b h
\end{array}\right.
$$

Using the fast control function (6) of the second order discrete system, paper[8] insteads the corresponding variables in rules (7-10), and constructs a kind of fast and precise differentiator with small phase delay and amplitude attenuation as below,

$$
\left\{\begin{array}{l}
{[u(k), b]=f a s t\left(x_{1}(k)-v(k), c_{1} x_{2}(k), r, c_{0} h\right)} \\
x_{1}(k+1)=x_{1}(k)+h \cdot x_{2}(k)+\frac{1}{2} r h^{2} \cdot u(k)-\frac{1}{3} b h^{3} \\
x_{2}(k+1)=x_{2}(k)+r h \cdot u(k)-\frac{1}{2} b h^{2}, k=0,1,2 \cdots
\end{array}\right.
$$

where, $x_{1}$ is the tracking signal of the input signal $v(k), x_{2}$ is the differential approximation signal of $v(k) . r$ is the speed coefficient, $h$ is the step, that is sample time. $c_{1}$ is the damping factor,$c_{0}$ is filtering factor. The component $x_{1}(k)$ of system (6) will be the fastest track of the input signal $v(k)_{[8] \text {. }}$

\section{Linearized Structure}

Generally $r$ is chosen large enough to make sure reach to the switch curve (2) in time $t_{A}(k)$. As $v(k)$ is changing, $M_{k}\left(x_{1}(k)-v(k), x_{2}(k)\right)$ can't reach to the switch curve (2) practically. Therefore, we only check whether $M_{k}\left(x_{1}(k)-v(k), x_{2}(k)\right)$ is in the switch curve, including the tracking ability of the differentiator when $t_{A}(k)<h$.

When $\Gamma\left(x_{1}, x_{2}, r\right) \neq 0$ and $t_{A}(k)<h$, choose the following precise control law as formula (8)),

$$
u(k)=-\left(-\frac{1}{2}+\frac{x_{2}(k) \cdot s(k)}{r h}+\frac{1}{2} \sqrt{\left.1+\frac{4}{r h}\left(x_{2}(k)+\frac{2\left(x_{1}(k)-v(k)\right)}{h}\right) s(k)\right)} \cdot s(k)\right.
$$


Generally $r$ is very large, (12) can be simplified as

$$
u(k) \doteq-\left(-\frac{1}{2}+\frac{x_{2}(k) s(k)}{r h}+\frac{1}{2}\left(1+\frac{1}{2}\left(\frac{4}{r h}\left(x_{2}(k)+\frac{2\left(x_{1}(k)-v(k)\right)}{h}\right) s(k)\right)\right)\right) \cdot s(k)=-\frac{2 x_{2}(k)}{r h}-\frac{2\left(x_{1}(k)-v(k)\right)}{r h^{2}}
$$

According to the fast control function $f a s t\left(x_{1}(k)-v(k), c_{1} x_{2}(k), r, c_{0} h\right)$ in paper[8], replacing the corresponding variables in formula(13) based on rules(7$10)$, that is $x_{2}(k) \rightarrow c_{1} x_{2}(k), h \rightarrow c_{0} h$, the other variables remain unchanged, we get

$$
u(k)=-\frac{2 x_{2}(k)}{r h}-\frac{2\left(x_{1}(k)-v(k)\right)}{r h^{2}}=-\frac{2 c_{1} x_{2}(k)}{r c_{0} h}-\frac{2\left(x_{1}(k)-v(k)\right)}{r c_{0}^{2} h^{2}}
$$

Substituting $b=0$ into formula (11), we have

$$
\left\{\begin{array}{l}
x_{1}(k+1)=x_{1}(k)+h \cdot x_{2}(k)+\frac{r h^{2} \cdot u(k)}{2} \\
x_{2}(k+1)=x_{2}(k)+r h \cdot u(k)
\end{array}\right.
$$

Substituting Eq.(14) into formula (15), we get the following linearization form of nonlinear differentiator(11)

$$
\left[\begin{array}{c}
x_{1}(k+1) \\
x_{2}(k+1)
\end{array}\right]=\left[\begin{array}{cc}
1-\frac{1}{c_{0}^{2}} & h\left(1-\frac{c_{1}}{c_{0}}\right) \\
-\frac{2}{c_{0}^{2} h} & 1-\frac{2 c_{1}}{c_{0}}
\end{array}\right]\left[\begin{array}{l}
x_{1}(k) \\
x_{2}(k)
\end{array}\right]+\frac{1}{c_{0}^{2}}\left[\begin{array}{c}
1 \\
\frac{2}{h}
\end{array}\right] v(k)
$$

\section{Filtering Ability Analysis}

As the sample step is generally small, system (15) can be approximated as

$$
\left\{\begin{array}{l}
\dot{x}_{1}=x_{2} \\
\dot{x}_{2}=u
\end{array}\right.
$$

where, $u=-\frac{2 c_{1} x_{2}}{c_{0} h}-\frac{2\left(x_{1}-v\right)}{c_{0}^{2} h^{2}}$.Suppose $v(k)=A \sin \omega t$, according to $x_{2}(k) \rightarrow c_{1} x_{2}(k), h \rightarrow c_{0} h,(17)$ is

$$
\ddot{x}_{1}+\frac{2 c_{1}}{c_{0} h} \dot{x}_{1}+\frac{2}{c_{0}^{2} h^{2}} x_{1}=\frac{2}{c_{0}^{2} h^{2}} v=\frac{2}{c_{0}^{2} h^{2}} A \sin \omega t
$$

Using the method of undetermined coefficients, the analytical solution of $x_{1}(t)$ is 


$$
x_{1}(t)=e^{-\frac{t}{h}}\left(C_{1} \cos \frac{t}{h}+C_{2} \sin \frac{t}{h}\right)-\frac{4 c_{1} c_{0} h \omega A}{c_{0}^{4} h^{4} \omega^{4}+4} \cos \omega t-\frac{2\left(c_{0}^{2} h^{2} \omega^{2}-2\right) A}{c_{0}^{4} h^{4} \omega^{4}+4} \sin \omega t
$$

when $\frac{t}{h}>4$, the first term including $e^{-\frac{t}{h}}$ in formula (19) will attenuate to 0 quickly.

According to the last two terms in formula (19), the stable solution of $x_{1}(t)$ is

$$
\bar{x}_{1}=-\frac{4 c_{1} c_{0} h \omega A}{c_{0}^{4} h^{4} \omega^{4}+4} \cos \omega t-\frac{2\left(c_{0}^{2} h^{2} \omega^{2}-2\right) A}{c_{0}^{4} h^{4} \omega^{4}+4} \sin \omega t=\beta A \sin (\omega t+\varphi)=\beta A \sin \omega(t+\tau)
$$

where, $\beta=\frac{2 \sqrt{c_{0}^{4} h^{4} \omega^{4}+4\left(c_{1}^{2}-1\right) c_{0}^{2} h^{2} \omega^{2}+4}}{c_{0}^{4} h^{4} \omega^{4}+4}$. The value of $\varphi$ can be discussed in two cases:

$$
\text { (1) when } 2-c_{0}^{2} h^{2} \omega^{2}>0, \varphi=-\tan ^{-1} \frac{2 c_{1} c_{0} h \omega}{2-c_{0}^{2} h^{2} \omega^{2}}
$$

(2) when $2-c_{0}^{2} h^{2} \omega^{2}<0, \varphi=\tan ^{-1} \frac{2 c_{1} c_{0} h \omega}{c_{0}^{2} h^{2} \omega^{2}-2}+\pi$

This paper mainly considers case (1), that is $2-c_{0}^{2} h^{2} \omega^{2}>0$.Suppose $c_{0} h \omega \ll \sqrt{2}$, we have

$$
\beta \doteq \sqrt{\left(c_{1}^{2}-1\right) c_{0}^{2} h^{2} \omega^{2}+1}, \tau \doteq-c_{1} c_{0} h
$$

Especially, when $c_{1}=1$, there is

$$
\beta \doteq 1, \tau \doteq-c_{0} h
$$

From the above formula , when $c_{0} h \omega \ll \sqrt{2}, c_{1}=1$, this filter will track input signal with small time delay $\tau=-c_{0} h$, and have hardly any amplitude loss.

\section{Restraining Disturbance Performance}

When disturbance $d(t)$ exists (where, $d(t)$ is bounded, that is $|d(t)| \leq h_{d}$ ), (17) can be changed as 


$$
\left\{\begin{array}{l}
\dot{x}_{1}=x_{2} \\
h^{2} \dot{x}_{2}=f\left(x_{1}-v(t)-d(t), h x_{2}\right)
\end{array}\right.
$$

where, $f\left(x_{1}-v(t)-d(t), \varepsilon x_{2}\right)=-\frac{2 c_{1} x_{2} h}{c_{0}}-\frac{2\left(x_{1}-v(t)-d(t)\right)}{c_{0}^{2}}$.

The error system of formula (25) and the derivatives of signal $v(t)$ is

$$
\left\{\begin{array}{l}
\dot{e}_{1}=e_{2} \\
h^{2} \ddot{e}_{2}=f\left(e_{1}-d(t), h e_{2}+h \frac{d v}{d t}\right)-h^{2} \frac{d^{2} v}{d t^{2}}
\end{array}\right.
$$

The above (26) can be changed as

$$
\left\{\begin{array}{l}
\frac{d e_{1}}{d t / h}=h e_{2} \\
\frac{d h e_{2}}{d t / h}=f\left(e_{1}-d(t), h e_{2}+h \frac{d v}{d t}\right)-h^{2} \frac{d^{2} v}{d t^{2}}
\end{array}\right.
$$

Let

$$
\tau=\frac{t}{h}, z_{1}(\tau)=e_{1}(t), z_{2}(\tau)=h e_{2}(t), z=\left[\begin{array}{ll}
z_{1} & z_{2}
\end{array}\right]^{T}, \bar{d}(\tau)=d(t)
$$

we get $z=\operatorname{diag}[1 \quad h] e$.According to formula (28), system(27) can be changed as

$$
\left\{\begin{array}{l}
\frac{d z_{1}}{d \tau}=z_{2} \\
\frac{d z_{2}}{d \tau}=f\left(z_{1}-\bar{d}(\tau), z_{2}+\frac{d v}{d \tau}\right)-\frac{d^{2} v}{d \tau^{2}}
\end{array}\right.
$$

Considering the following system [9-10]

$$
\left\{\begin{array}{l}
\frac{d \tilde{z}_{1}}{d t}=\tilde{z}_{2} \\
\frac{d \tilde{z}_{2}}{d t}=f\left(\tilde{z}_{1}, \tilde{z}_{2}\right)
\end{array}\right.
$$

Suppose the equilibrium point of this system is stable in finite time, and the settling time function $T_{f}$ is continuous in the original point, where $f\left(\tilde{z}_{1}, \tilde{z}_{2}\right)$ is continuous and $f(0)=0$. As seen, formula (29) is the perturbed system of system (30). 
Choose the Lyapunov function $(V \circ z)(\tau)$ which is Lipschitz continuous and the Lipschitz constant is $M$. Then, the upper right derivative of $(V \circ z)(\tau)$ is [910,13-14]

$$
D^{+}(V \circ z)(\tau)=\frac{\partial V}{\partial z}(z)\left[z_{2} \quad f\left(z_{1}, z_{2}+\frac{d v}{d \tau}\right)-\frac{d^{2} v}{d \tau^{2}}\right]^{T} \leq \dot{V}+M\left(\bar{a} h_{d}^{\rho_{d}}+\bar{a} h^{\rho_{1}} h_{1}^{\rho_{1}}+h_{2} h^{2}\right)
$$

where, $h_{1}, h_{2}$ is the upper bound of the first and the second derivatives of signal $v(t)$ respectively. Suppose $\rho_{d} \in(0,1]$ and $\delta=\bar{a} h^{\rho_{1}} h_{1}^{\rho_{1}}+h_{2}$, from formula(31), we get

$$
D^{+}(V \circ z)(\tau) \leq \dot{V}+M \bar{a} h_{d}^{\rho_{d}}+h^{\rho} M \delta
$$

For the practical system, there is a constant $T>0\left(T>T_{f}\right)$,for $t_{j-1} / h+T\left(z\left(t_{j-1} / h\right)\right) \leq \tau<t_{j} / h, \mathrm{j}=1, \ldots, k+1$, which satisfied

$$
\|z(\tau)\| \leq \frac{(V(z(\tau)))^{1-\theta}}{r c(1-\theta)} \leq \frac{\left(\frac{2 M \bar{a} h_{d}^{\rho_{d}}+2 h^{\rho} M \delta}{c}\right)^{\frac{1-\theta}{\theta}}}{r c(1-\theta)}
$$

where, $\theta \in(0,0.5), r=\frac{1-\theta}{\theta}, c>1$.

As $\theta$ can be chosen small enough, $\frac{1-\theta}{\theta}$ can become large enough.

$$
\frac{2 M \bar{a} h_{d}^{\rho_{d}}+2 h^{\rho} M \delta}{c}<1
$$

From formula (34), we have

$$
h<\left(\frac{h}{2 M \delta}\right)^{\frac{1}{\rho}}, h_{d}<\left(\frac{c-2 h^{\rho} M \delta}{2 M \bar{a}}\right)^{\frac{1}{\rho_{d}}}
$$

At this moment, the noise of system (25) can be restrained effectively.

\section{Conclusions}

This paper constructs a second order linear differentiator with small phase delay and amplitude attestation using the optimal fast synthesis function of double integral series system. Using the discrete linear structure, we analyze the tracking and filtering ability of this differentiator in time domain, and discuss the 
restraining disturbance performance of system to boundary disturbance based on the optimal theory and singular perturbation technique.

\section{Acknowledgment}

This work was supported by National Natural Science Foundation of China (61304036) and Natural Science Foundation of Hunan Province (2015JJ3019), and China Postdoctoral Science Foundation (2014M562651, 2015T81132).

\section{References}

[1]. J.Q. Han. From PID to Active Disturbance Rejection Control, IEEE Transactions on Industrial Electronics. 56(3)(2009) 900-906.

[2]. S.P. Bhat, D.S. Bernstein. Continuous, finite-time stabilization of the translational and rotational double integrators, IEEE Trans. Automat. Control. 43 (1998) 678-682.

[3]. S.P. Bhat, D.S. Bernstein. Example of indeterminacy in classical dynamics, Internat. J. Theoret. Phys.. 36,(1997)545-550.

[4]. H. Khalil. Robust servomechnasim output feedback controller for feedback linearizable systems, Automatica. 30(1994) 1587-1599.

[5]. X. Wang, Z. Chen, Z.Yuan. Design and analysis for new discrete trackingdifferentiators, Applied Mathmatic Transactions for Universities. Series B, 18 (2) (,2003) 214-222.

[6]. A. Levant. Robust exact differentiation via sliding mode technique, Automatica. 34, 1998:379-384.

[7]. A. Levant. High order sliding modes, differentiation and output feedback control. Int. J. Contr.. 76(9/10)(2003) 924-941.

[8]. Y.D. Xie, Z.Q. Long, J. Li. Analysis of the second-order nonlinear discretetime tracking-differentiator as a filtering, Proceeding of the 8th WCICA. (2007) Chengdu, China:6750-6755.

[9]. D. Zhang , Y.G. Li, H.K. Liu et al.. Restrain the effects of vehicleguideway dynamic interaction: bandstop filter method, Maglev2008 Proceedings. (2008)

[10]. H. Li, R.M. Goodall. Linear and non-linear skyhook damping control laws for active railway suspensions, Control Eng. Pract.. 7 (7),(1999) 843-850.

[11]. J.Q. Han. From PID to active disturbance rejection control, IEEE Trans. Ind. Electron.. 56(3),(2009)900-906.

[12]. L.L. Zhang, Z.Z. Zhang, L.H. Huang. Hybrid nonlinear differentiator design for Permanent-electro magnetic suspension maglev system, Signal Processing. 6(6) (2012)559-567. 
[13]. S.P. Bhat, D.S. Bernstein. Finite-time stability of continuous autonomous systems, SIAM J. Control Optim.. 38(3)(2000)751-766.

[14]. S.P. Bhat, D.S. Bernstein. Lyapunov Analysis of Finite-Time Diferential Equations, Proceedings of the American Control Conference, Seattle, WA. (1995)1831-1832. 